EESTI NSV TEADUSTE AKADEEMIA TOIMETISED. 28. KOIDE FOOSIKA * MATEMAATIKA. 1979, NR. 1

ИЗВЕСТИЯ АКАДЕМИИ НАУК ЭСТОНСКОН ССР. ТОМ 28 ФИЗИКА * МАТЕМАТИКА, 1979, 스 1

удК $\quad 621.316 .72 .001 .5$

Maйpe $О Я В Е Э Р$, В. САРВ

\title{
ОБМЕН ЭНЕРГИИ МЕЖДУ ГАРМОНИКАМИ В КЛЮЧАХ РЕГУЛЯТОРА ПЕРЕМЕННОГО НАПРЯЖЕНИЯ ПРИ ВЫСОКОЧАСТОТНОЙ ШИРОТНО-ИМПУЛЬСНОЙ модуляции. І
}

\author{
(Представлена И. Эпиком)
}

Совершенствование ключевых преобразователей параметров электроэнергии тесно связано с поиском подходящих способов и целесообразных технических приемов улучшения спектрального состава входного и выходного токов. В цепях, в которых основными преобразовательными элементами, непосредственно участвующими в обмене энергии, являются ключи, ток и напряжение последних наряду с полезными гармониками обязательно содержат и искажающие [ $\left.{ }^{1}\right]$. Поэтому улучшение качества работы таких преобразователей сводится к минимизации и локализации искажающих гармоник тока и напряжения ключей, поскольку устранить их совсем не представляется возможным. Иногда, например в трансформаторно-ключевых дискретных регуляторах переменного напряжения, в которых основными преобразовательными элементами являются не ключи, а трансформаторы, искажения полностью устранимы. В этом случае полная мощность ключей в установившемся режиме равняется нулю, т. е. они в обмене энергии не участвуют.

Одним из перспективных способов регулирования переменного напряжения является импульсная модуляция $\left[{ }^{2-5}\right]$. В импульсных регуляторах переменного напряжения полная мощность ключей как основных преобразовательных элементов, участвующих в обмене энергии, отличается от нуля $\left[{ }^{6}\right]$ и поэтому в ходе регулирования их ток и напряжение неизбежно искажаются. При этом возможности минимизации и локализации искажений существенно зависят от энергетического процесса в самом ключе, в частности от характера обмена энергии между гармониками.

Проанализируем основные частотно-энергетические соотношения в схеме широтно-импульсного регулирования переменного напряжения (рис. $1, a)$, предполагая, что напряжение питания $u=U_{m} \sin \omega t$, ключи I и II идеальны, последовательно с нагрузкой $z$ соединен идеальный полосовой фильтр $F(\omega)$, пропускающий только основную гармонику с круговой частотой $\omega$, круговая частота модуляции $\Omega>2 \omega$, т. е., согласно классификации в $\left[{ }^{4}\right]$, ограничимся случаем высокочастотной модуляции.

В основе любого способа улучшения спектрального состава токов ключевого преобразователя лежит совершенствование обмена энергии между источником питания, нагрузкой, ключами и фильтрующими эле- 
\%

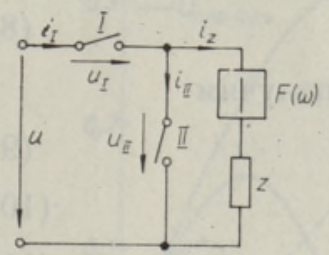

Рис, 1 , $\delta$

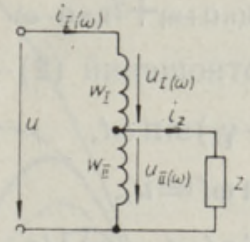

Рис. 2.
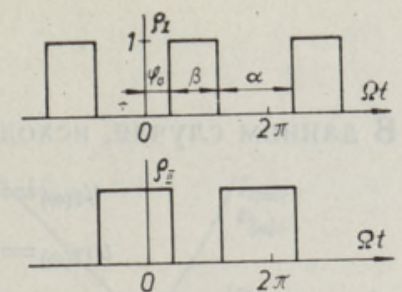

ментами. Когда нагрузка и фильтрующие элементы линейны, генерирование искажающих гармоник происходит только в ключах. Хотя при этом мгновенная мощность каждого идеального ключа равна нулю, мгновенные мощности отдельных гармоник могут отличаться от нуля, в результате чего в ключах возможен обмен энергии между гармониками $\left[{ }^{7}\right]$.

В цепях преобразования частоты, выпрямления и инвертирования ключи потребляют энергию на частоте питания и генерируют ее преимущественно на выходной частоте. Хотя в регуляторах переменного напряжения входная и выходная частоты равны, ключи могут развивать активную мощность как на основной, так и на искажающих частотах.

Для определения частотно-энергетических соотношений в схеме на рис. 1,a выпишем выражения коммутационных функций ключей I и II согласно рис. 2 :

$$
\begin{gathered}
\text { QI }(\Omega t)=\beta / 2 \pi+\sum_{n=1}^{\infty}\left[2 \sin (n \beta / 2) \cos n\left(\Omega t-\beta / 2-\varphi_{0}\right)\right] / \pi n, \\
\text { QII }(\Omega t)=1-\mathrm{QI}(\Omega t),
\end{gathered}
$$

где $\beta$ - угол, соответствующий времени открытого состояния ключа I и определяющий его скважность $\gamma_{\mathrm{I}}=\beta / 2 \pi ; \gamma_{\mathrm{II}}=\alpha / 2 \pi=(2 \pi-\beta) / 2 \pi=$ $=1-\gamma_{\mathrm{I}} ; \varphi_{0}-$ начальная фаза; $n=1,2,3, \ldots \infty$. Для мгновенных значений напряжений и токов ключей I и II на рис. 1, $a$ действительны соотношения

$$
u_{\mathrm{I}}=u_{\varrho \mathrm{II}} ; \quad i_{\mathrm{I}}=i_{z} \mathrm{I} ; \quad u_{\mathrm{II}}=u_{\mathrm{QI}} ; \quad i_{\mathrm{II}}=-i_{z} \mathrm{\varrho II}_{\mathrm{II}}
$$

где $i_{z}$ - мгновенное значение тока нагрузки, равное

$$
i_{z}=\left[U_{m} \gamma_{\mathrm{I}} \sin (\omega t-\varphi)\right] / z,
$$

и $甲$ - его фазовый сдвиг.

Для определения активных и реактивных мощностей всех элементов схемы целесообразно записать мгновенные значения токов и напряжений ключа I в следующем виде (выражения для ключа II аналогичны):

$$
\begin{aligned}
& u_{\mathrm{I}}=u_{\mathrm{I}(\omega)}+\sum_{n=1}^{\infty} u_{\mathrm{I} n}, \\
& i_{\mathrm{I}}=i_{\mathrm{I}(\omega)}+\sum_{n=1}^{\infty} i_{\mathrm{I} n} .
\end{aligned}
$$

Первый член приведенных выражений - это только основная гармоника тока или напряжения ключа, второй - гармоники верхних $n \Omega+\omega$ и нижних $n \Omega-\omega$ боковых частот. Например, 


$$
\begin{gathered}
u_{\mathrm{I} n}=u_{\mathrm{I}(n \Omega+\omega)}+u_{\mathrm{I}(n \Omega-\omega)}, \\
i_{\mathrm{I} n}=i_{\mathrm{I}(n \Omega+\omega)}+i_{\mathrm{I}(n \Omega-\omega)} .
\end{gathered}
$$

В данном случае, исходя из соотношений $(2)-(4)$, получим

$$
\begin{gathered}
u_{\mathrm{I}(\omega)}=U_{m}\left(1-\gamma_{\mathrm{I}}\right) \sin \omega t, \\
u_{\mathrm{II}(\omega)}=U_{m} \gamma_{\mathrm{I}} \sin \omega t=u_{z}, \\
i_{\mathrm{I}(\omega)}=\left[U_{m} \gamma_{\mathrm{I}}^{2} \sin (\omega t-\varphi)\right] / z, \\
i_{\mathrm{II}(\omega)}=-\left[U_{m} \gamma_{\mathrm{I}}\left(1-\gamma_{\mathrm{I}}\right) \sin (\omega t-\varphi)\right] / z, \\
u_{\mathrm{I} n}=-u_{\mathrm{II} n}=-U_{m} \sin n \gamma_{\mathrm{I}} \pi\left\{\sin \left[(n \Omega+\omega) t-n \gamma_{\mathrm{I}} \pi-n \varphi_{0}\right]-\right. \\
\left.-\sin \left[(n \Omega-\omega) t-n \gamma_{\mathrm{I}} \pi-n \varphi_{0}\right]\right\} / \pi n, \\
i_{\mathrm{I} n}=i_{\mathrm{II} n}=U_{m} \gamma_{\mathrm{I}} \sin n \gamma_{\mathrm{I}} \pi\left\{\sin \left[(n \Omega+\omega) t-n \gamma_{\mathrm{I}} \pi-n \varphi_{0}-\varphi\right]-\right. \\
\left.-\sin \left[(n \Omega-\omega) t-n \gamma_{\mathrm{I}} \pi-n \varphi_{0}+\varphi\right]\right\} / \pi n z .
\end{gathered}
$$

Активная мощность, потребляемая от источника питания, равняется активной мощности нагрузки и выражается в виде

$$
P_{(\omega)}=P_{z}=\left[U_{m}^{2} \gamma_{\mathrm{I}}^{2} \cos \varphi\right] / 2 z .
$$

Активная и реактивная мощности основной гармоники и боковых частот ключей I и II имеют вид

$$
\begin{aligned}
& P_{\mathrm{I}(\omega)}=\left[U_{m}^{2} \gamma_{\mathrm{I}}^{2}\left(1-\gamma_{\mathrm{I}}\right) \cos \varphi\right] / 2 z=-P_{\mathrm{II}(\omega),} \\
& Q_{\mathrm{I}(\omega)}=\left[U_{m}^{2} \gamma_{\mathrm{I}}^{2}\left(1-\gamma_{\mathrm{I}}\right) \sin \varphi\right] / 2 z=-Q_{\mathrm{II}(\omega),} \\
& P_{\mathrm{I}(n \Omega+\omega)}=-\left[U_{m}^{2} \gamma_{\mathrm{I}} \sin ^{2} n \gamma_{\mathrm{I}} \pi \cos \varphi\right] / 2 \pi^{2} n^{2} z, \\
& Q_{\mathrm{I}(n \Omega+\omega)}=-\left[U_{m}^{2} \gamma_{\mathrm{I}} \sin ^{2} n \gamma_{\mathrm{I}} \pi \sin \varphi\right] / 2 \pi^{2} n^{2} z .
\end{aligned}
$$

Из анализа следует, что

$$
\begin{aligned}
& P_{\mathrm{I}(n \Omega+\omega)}=P_{\mathrm{I}(n \Omega-\omega)}=-P_{\mathrm{II}(n \Omega+\omega)}=-P_{\mathrm{II}(n \Omega-\omega)}, \\
& Q_{\mathrm{I}(n \Omega+\omega)}=-Q_{\mathrm{I}(n \Omega-\omega)}=-Q_{\mathrm{II}(n \Omega+\omega)}=Q_{\mathrm{II}(n \Omega-\omega)} .
\end{aligned}
$$

По аналогии с (7) и (8) обозначим:

$$
\begin{aligned}
& P_{\mathrm{I} n}=P_{\mathrm{I}(n \Omega+\omega)}+P_{\mathrm{I}(n \Omega-\omega)}, \\
& Q_{\mathrm{I} n}=Q_{\mathrm{I}(n \Omega+\omega)}+Q_{\mathrm{I}(n \Omega-\omega))} .
\end{aligned}
$$

Тогда

$$
\begin{gathered}
\sum_{n=1}^{\infty} P_{\mathrm{I} n}=-\sum_{n=1}^{\infty} P_{\mathrm{II} n}, \\
Q_{\mathrm{I} n}=Q_{\amalg I n}=0 .
\end{gathered}
$$

Так как активная мощность ключа равняется нулю, то

$$
\sum_{n=1}^{\infty} P_{\mathrm{I} n}+P_{\mathrm{I}(\omega)}=0 ; \quad \sum_{n=1}^{\infty} P_{\mathrm{II} n}+P_{\mathrm{II}(\omega)}=0 .
$$

Приведенные соотношения показывают, что ключи схемы участвуют в процессе обмена энергии следующим образом: ключ I потребляет энергию на основной частоте с мощностью $P_{\mathrm{I}(\omega)}$ и генерирует ее на 


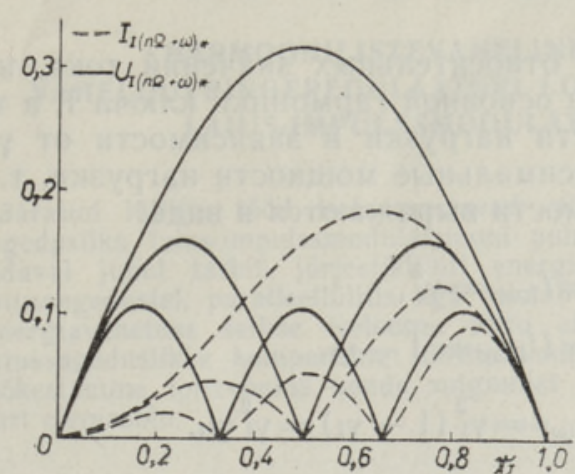

Рис. 3

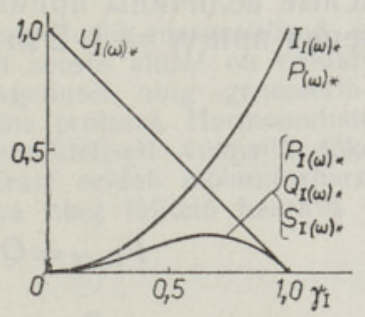

Рис. 4.

боковых частотах $n \Omega+\omega$ и $n \Omega-\omega$ с мощностью $\sum_{n=1}^{\infty} P_{\text {In }} ;$ ключ II, наоборот, потребляет энергию на боковых частотах $\left(\sum_{n=1}^{\infty} P_{\operatorname{IIn} n}\right)$ и генерирует ее на основной частоте $\left(P_{I I(\omega)}\right)$.

Реактивные мощности источника питания и нагрузки равны $\left(Q_{(\omega)}=Q_{z}\right)$. Реактивные мощности первой гармоники обоих ключей взаимно компенсируются, а боковых, искажающих, гармоник уравновешиваются в каждом ключе отдельно.

Интересно отметить, что передача энергии основной гармоники от источника питания в нагрузку в схеме ключевого преобразователя (рис. $1, a)$ происходит так же, как в схеме автотрансформатора (рис. $1, \sigma)\left[{ }^{5}\right]$, только функции ключей I и II в первой выполняют обмотки $w_{\text {I и }} w_{\text {II }}$ во второй. Энергетическая связь между обмотками обеспечивается единым для них магнитным потоком (рис. 1,6$)$, а между ключами I и II - единым током искажающих гармоник (рис. 1,a):

$$
\sum_{n=1}^{\infty} i_{\mathrm{I} n}=\sum_{n=1}^{\infty} i_{\mathrm{II} n}
$$

Это наглядно показывает, что наличие контура замыкания тока искажающих гармоник в регуляторе переменного напряжения при высокочастотной широтно-импульсной модуляции является необходимым условием для его работы.

На рис. 3 показаны зависимости относительных значений напряжения и тока боковых частот ключа I

$$
\begin{aligned}
& U_{\mathrm{I}(n \Omega+\omega) m^{*}}=\left(\sin n \gamma_{\mathrm{I}} \pi\right) / \pi n, \\
& I_{\mathrm{I}(n \Omega+\omega) m^{*}}=\left(\gamma_{\mathrm{I}} \sin n \gamma_{\mathrm{I}} \pi\right) / \pi n
\end{aligned}
$$

от тех же значений нагрузки $U_{z m^{*}}=I_{z m^{*}}=\gamma_{\mathrm{I}}$ при $n=1,2$, 3. Базисными величинами выбраны максимальные амплитудные значения тока и напряжения нагрузки, т. е. их значения при $\gamma_{\mathrm{I}}=1$.

Используя соответствующие действующие значения, можем записать:

$$
\begin{gathered}
U_{\mathrm{I}(n \Omega+\omega) m^{*}}=U_{\mathrm{I}(n \Omega+\omega)^{*}}, \\
I_{\mathrm{I}(n \Omega+\omega) m^{*}}=I_{\mathrm{I}(n \Omega+\omega)^{*}}, \\
I_{z m^{*}}=I_{z^{*}}=U_{z m^{*}}=U_{z^{*}} .
\end{gathered}
$$


Рис. 4 иллюстрирует изменение относительных значений тока, напряжения и составляющих мощности основной гармоники ключа I, а также относительной активной мощности нагрузки в зависимости от $\gamma_{\mathrm{I}}$. За базисные величины приняты максимальные мощности нагрузки, т. е. их значения при $\gamma_{\mathrm{I}}=1$. Эти зависимости выражаются в виде:

$$
\begin{gathered}
I_{\mathrm{I}(\omega) m^{*}}=I_{\mathrm{I}(\omega)^{*}}=\gamma_{\mathrm{I}}^{2}, \\
U_{\mathrm{I}(\omega) m^{*}}=U_{\mathrm{I}(\omega)^{*}}=1-\gamma_{\mathrm{I}}, \\
P_{\mathrm{I}(\omega)^{*}}=Q_{\mathrm{I}(\omega)^{*}}=S_{\mathrm{I}(\omega)^{*}}=\gamma_{\mathrm{I}}^{2}\left(1-\gamma_{\mathrm{I}}\right)=\gamma_{\mathrm{I}}^{2} \gamma_{\mathrm{II}}, \\
P_{(\omega)^{*}}=P_{z^{*}}=\gamma_{\mathrm{I}}^{2} .
\end{gathered}
$$

Соотношения $(28),(29),(33)-(36)$ не зависят от фазового сдвига тока нагрузки $\varphi$.

Итак, из анализа работы ключевого регулятора переменного напряжения, работающего в режиме высокочастотной широтно-импульсной модуляции и подавления искажающих гармоник в токе нагрузки, можно заключить следующее.

1. Полная, активная и реактивная мощности основной гармоники ключей пропорциональны соответствующим мощностям нагрузки; коэффициент пропорциональности равен скважности модуляции параллельного ключа.

2. В последовательном ключе потребление энергии основной гармоники уравновешивается генерированием энергии искажающих гармоник, а в параллельном ключе генерирование энергии основной гармоники - потреблением энергии искажающих гармоник.

3. Реактивные мощности основной гармоники последовательного и параллельного ключей компенсируются взаимно, а реактивные мощности искажающих гармоник - в каждом ключе отдельно.

4. По передаче энергии основной гармоники от источника питания в нагрузку схема высокочастотного широтно-импульсного регулятора переменного напряжения аналогична схеме автотрансформатора.

5. Заграждение искажающих гармоник в токе питания возможно только при наличии контура замыкания этих гармоник.

$$
\text { Л ИТЕРАТУ Р А }
$$

1. О я в еэ р М., Саккос Т., С ар в В., Вентильно-индуктивные цепи непрерывного амплитудного регулирования переменного напряжения, Таллин, «Валгус»,

1978. Emanuel-Eigeles, A., Appelbaum, J., Electron. Lett., 4, № 2, 26-28 (1968).

3. Emanuel-Eigeles, A., Appelbaum, J., Arch. Elektrotechn., 53, № 5, 326-336 (1970).

4. Гел льм н М. В., Лохов С. П., Тиристорные регуляторы переменного напряжения, М., «Энергия», 1975.

5. Жар.ский Б. К., Голу бев В. В., Препринт-96 ИЭд АН УССР, Киев, 1975.

6. М илях А. Н., Ж а р ски й Б. К., В сб.: Проблемы технической электродинамики, вып. 62 , Киев, «Наук. думка», 1977, с. 3-7.

7. О я в е э р М., С а р в В., Изв. АН ЭССР, Физ. Матем., 24, № 1, 78-80 (1975).

Ннститут термофизики и электрофизики Академии наук Эстонской ССР
Поступила в редакцию 28/VIII 1978 


\title{
HARMOONILISTEVAHELINE ENERGIAVAHETUS VAHELDUVPINGEREGULAATORI LULITITES KORGSAGEDUSLIKU LAIUS-IMPULSSMODULATSIOONI KORRAL. I
}

On määratud lülitite tööd iseloomustavad põhilised sageduslik-energeetilised seosed kõrgsagedusliku laius-impulssmodulatsiooni puhul. Leitud seoste alusel on näidatud, et vaadeldaval juhul tarbib järjestiklüliti energiat toitesagedusel ning genereerib seda moonutussagedustel, paralleellülitis aga toimub vastupidine protsess. Harmoonilistevahelise energiavahetuse sellise iseloomu tōttu ei ole pōhimōtteliselt vōimalik tōkestada moonutussageduslikke komponente lülitite voolus. Seepärast eeldab moonutusharmooniliste tōkestamine toitevoolus nende sulgumist võimaldava ning lüliteid haarava voolukontuuri olemasolu.

\author{
Maire OJAVEER, V. SARV
}

\section{ENERGY EXCHANGE BETWEEN HARMONICS IN SWITCHES OF ALTERNATING VOLTAGE REGULATOR AT HIGH-FREQUENCY PULSE-WIDTH MODULATION}

Frequency-power relationships in an ideal alternating voltage switched regulator operating in the mode of high-frequency pulse-width modulation and suppressed distortion harmonics in the load current are analysed. As the principal converting elements in the regulator are switches, the current and voltage are inevitably distorted and therefore apparent power of the switch differs from zero. Since the total active power of an ideal switch is zero, power consumption at the fundamental harmonic in the series switch is equal to power generation at distortion harmonics; power consumption at distortion harmonics in the parallel switch equals to power generation at the fundamental harmonic. The sum of reactive powers at the fundamental harmonic is zero in both switches combined; the sum of reactive powers at distortion harmonics is zero in both switches separately. Apparent, active and reactive powers at the fundamental harmonic in the switches are proportional to the same powers in the load.

From the point of view of the fundamental harmonic power transmission from a-c source to the load, the regulator under consideration is analogous to auto-transiormer.

The equations and curves presented in the paper show per unit values of both distortion harmonics voltage and current and the fundamental harmonic powers in the switch as plotted against per unit values of the load voltage and current, both of which being equal to the duty ratio of the series switch. 